\title{
DISCOVERING MATERIAL RECOVERY SCENARIOS FOR INDUSTRIAL MACHINERY: A CASE BASED APPROACH
}

\author{
William Z. Bernstein \\ Mechanical Engineering, Purdue University \\ West Lafayette, IN, USA
}

\author{
Devarajan Ramanujan \\ Mechanical Engineering, Purdue University \\ West Lafayette, IN, USA
}

\author{
Mikko Koho \\ Production Engineering, \\ Tampere University of \\ Technology \\ Tampere, Finland
}

\author{
Fu Zhao \\ Mechanical Engineering \\ Division of Environmental and \\ Ecological Engineering, Purdue \\ University \\ West Lafayette, IN, USA
}

\author{
Karthik Ramani \\ Mechanical Engineering, Purdue \\ University \\ West Lafayette, IN, USA
}

\begin{abstract}
Decision-making methodologies for evaluating a product's end-of-life options have become a significant area of research. Extensive work has been carried out in the area of product recovery, e.g. module-based disassemblability, reverse logistics, remanufacturing, material recyclability, among others. Some of these methods use graphical representations in the form of disassembly trees and/or networks to find feasible solutions with computational approaches, but have not been made applicable to larger, more complex electrohydraulic mechanical systems. The work presented in this paper aims to apply a disassembly assessment technique by comparing a component's disassembly effort to a reward such as recycling value or energy recovery from recycling. First, the disassembly network is represented by a directed graph where weighted edges represent reward/cost. Next, an implementation of Dijkstra's algorithm is used to compute the optimal disassembly path that minimizes the sum of the edge weights. Lastly, the optimal disassembly paths for each individual reward are compared to discover the globally optimal disassembly scenario. This method is applied to a real-world case study of an underground mining drill rig with direct contributions from engineers involved in the development of the machine itself. Specific component recovery options are recommended based on the methodology and alternative design practices are suggested to improve product recyclability.
\end{abstract}

Keywords: Design of Environment, EOL options, Material recycling, Network Analysis

\section{INTRODUCTION}

Growing public concern regarding issues related to environmental sustainability is currently motivating a paradigm shift in design thinking within organizations [1]. There is an imminent need to equip industrial manufacturers with novel design infrastructure in the form of easy-to-use tools that predict a product's net environmental footprint across its entire lifecycle (i.e. from material acquisition through its eventual disposal). Decision-making methodologies for evaluating a product's end-of-life (EOL) options have become a significant area of research in light of these new requirements. Component affinities for EOL options (i.e. reuse, recycling, remanufacturing and disposal) have been developed and studied with respect to their industrial relevance. Developing, for instance, a remanufacturing affinity for components and modules is challenging due to downstream uncertainties involved in EOL product quality affected by many factors associated with the use of a product, e.g. cyclic loading and structural fatigue. These uncertainties, nonetheless, have not halted ongoing work in this area due to its ever-increasing importance.

Extensive research has been hence focused on the area of product recovery, e.g. module-based disassemblability, reverse logistics, remanufacturing, material recyclability, among others. Methods for product-specific disassembly planning have proven to greatly influence the nature of this research space. Many methods use graphical representations in the form of disassembly trees and/or networks to find feasible solutions with computational approaches. However, most of the published work has focused on assessing the disassemblability of simple modular products or high-volume electronic products 
such as personal computer (PC) towers. Though important, these methods are often inapplicable to larger and more complex electrohydraulic mechanical systems such as automobiles, earth moving equipment and industrial machine tools due to problems associated with variations in product architecture, disassembly tasks and design methodologies. Many studies have concentrated on developing cost models for disassembly scenarios of products [2]. Some of these methodologies have included ecological benefits for certain pathways [3]. Others have included material sequestration value estimations [4]. These proposed methodologies have often been implemented within case studies in order to validate their efficacy and breadth, but again there is limited penetrance in similar assessments for industries related to complex heavy machinery.

For developing initial takeback and recycling plans for such projects, it is important for every input phase of the analysis to be quick and easy-to-use, especially if the firm utilizing the method wishes to asses several different design configurations. For example, an automobile may contain some 5000 components for which an extensive life cycle assessment (LCA) would be a cost and time intensive undertaking [5]. Added costs associated with LCA could be unjustified when dealing with EOL decision making, when specific subassemblies or components dominate a product's total ecological footprint. Hence, a useful assessment methodology should include appropriate metrics (i.e. disassemblability measurement, embodied energy, $\mathrm{CO} 2$ footprint, recycling value) with product-specific rigor so as to efficiently commit resources while continuing to meet organizational business goals.

The work presented in this paper aims to apply a disassembly assessment technique by comparing a component's disassembly effort to a reward such as recycling value or energy recovery from recycling. First, the disassembly network is represented by a directed graph where weighted edges represent reward/cost. Next, an implementation of Dijkstra's algorithm is used to compute the optimal disassembly path that minimizes the sum of the edge weights. Lastly, the optimal disassembly paths for each individual cost, or the inverse of reward, are compared to discover the globally optimal disassembly scenario. This method is applied to a real-world case study of an underground mining drill rig with direct contributions from engineers involved in the design of the machine itself. Specific component recovery options are recommended based on the methodology and alternative design practices are suggested to improve product recyclability.

The specific case study, for which the presented methodology is applied, is a dual-boom jumbo earth-moving machine. The machine consists of multiple complex subassemblies (e.g. engine block, drilling module, multiple hydraulic systems) that require energy-intensive materials to meet its specialized characteristics. As of now, no specific recycling or disassembly instructions are provided to the user. As a result, in many cases, the mining rigs are simply left underground after their useful life in remote locations across the globe. The significant opportunity for material sequestration motivated the machine's company to begin initial plans for component recovery. The project goal is to discover targeted disassembly pathways for significant reward in terms of material, embodied energy and recycling value at a reasonable disassembly cost.

Figure 1 outlines the general proposed methodology. The tasks include (1) constructing a product-specific graph representing the disassembly pathways from the parent module, (2) retrieving qualitative, subjective disassemblability scores for disassembly pathways, (3) estimating embodied energy, $\mathrm{CO} 2$ footprint, and recycling recovery value based on a bill of materials (BOM), (4) combining all first three steps to create a weighted directed graph, and (5) traversing the directed graph through Dijkstra's shortest path algorithm and discovering feasible recovery options.

\section{BRIEF LITERATURE REVIEW}

Work has already been conducted on developing disassembly plans based on end-of-life value, e.g. [6], in order to make existing methods applicable during early design. Others have focused on solving the product recovery problem with network algorithm using optimizing cost based on disassembly metrics and recovery value, e.g. [7-9]. These methods usually require information that is rather difficult to retrieve without running time-extensive validation testing. From a different perspective, some papers have focused on qualitative disassembly to add relevance for such analysis to the design phase. Kroll and Hanft utilize a disassembly evaluation chart to assess each disassembly criterion via a qualitative

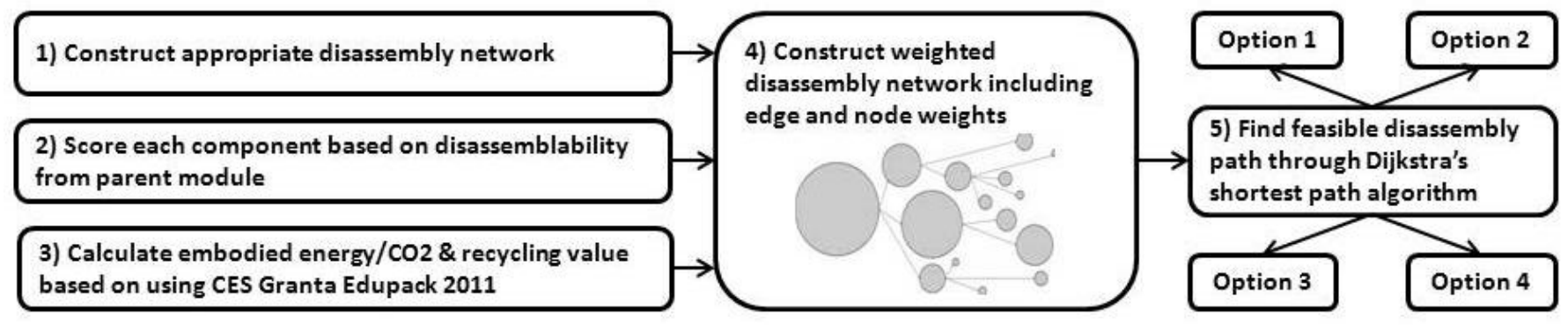

Figure 1: Schematic of overall proposed methodology. 
measure, then use quantitative methods to retrieve the most viable disassembly process [10]. Das et al. developed a qualitative scoring system through seven weighted factors to provide a normalized disassembly score focusing on analyzing the disassembly of PC towers [11].

Recently, researchers within the area of disassembly science have attempted to connect embedded lifecycle assessment (LCA) data within disassembly networking. Kuo 2006 conducted lifecycle and disassembly analyses for a rollerblade design that were both utilized to build a knowledge network of the used product. This paper used an undirected graph to represent the disassembly process based on separating modules [12]. Alternatively, Petri nets have been implemented in order to optimize disassembly planning while incorporating required remanufacturing operations [13]. Another group used computer-aided design (CAD) features to perform a scatter search to determine a total disassembly cost [14]. Shrivastava et al. developed a GUI that supports decision making for material recovery based on disassemblability, recyclability, profitability, and environmental score impact for electronic products [15]. In addition, Yi et al. coined a new method, D-L-N modeling, in which recycling strategies are optimized by using a productspecific hierarchical structure [16].

The before-mentioned work is specifically centered on modular products that have relatively simple disassembly characteristics. For heavy machinery, unless the system is returned to the assembly plant, it is unfeasible to disassemble large modules at a time due to a number of reasons, e.g. the remote locations of the machines, absence of heavy and capable tools, and lack of product expertise of the user.

\section{METHODOLOGY}

There have been many models published as mentioned above that estimate the ease of disassembly of components, subassemblies, or even an entire product. Most of them are focused on the mass disassembly of old PC towers and highvolume, highly modular products. Some are very precise, requiring specific disassembly times, associated costs, and other detailed information. Others are rather subjective in nature based on qualitative ranking criteria to retrueve a quick, but less reliable disassemblability score. In practice, the detailed methodologies are applicable to cost-benefit analysis of remanufacturing and other post production assessments, while the qualitative scoring sheets are used within the design phase, in which the ambiguity of specifications is great. Due to the brevity of this specific project (i.e. 3 months), a qualitative assessment tool to rank disassemblability [11] of each subassembly was chosen. Additionally, gathering precise disassembly data necessary for other quantitative methods is cost and time intensive.

In order to identify the disassembly steps for which require disassemblability scores, a disassembly network is constructed with input from project stakeholders (e.g. assembly workers, design team members).

\section{Disassembly Network Development}

The mining rig studied is assembled in a modular fashion, in which each high-level module is afforded its own assembly line and later converge to a final assembly line. It is unfeasible, however, to disassemble the same modules in order to recover specific components due to the machines' remote locations and the difficulties associated with each disassembly step, in terms of necessary tools, applied force, and worker expertise. As a result, the disassembly network must be developed on a component basis rather than through modulerelated interdependencies. Similar to other complex products, structure-to-structure dependencies within the mining rigs is highly coupled, multi-layered, and rather complex. In this study, 60 of the rig's components/subassemblies were studied.

The disassembly network is first heuristically constructed in graphical form, then stakeholders within the project can check the network in terms of its fidelity and accuracy. The network is converted to an adjacency matrix for which a " 1 " within entry $(a, b)$ denotes that disassembling component $a$ will enable the recovery of component $b$. The resulting sparse matrix allows for various clustering, banding, and sequencing techniques to be performed. These well-known methods are available from DSMweb.org [17]. This matrix represents a Design Structure Matrix (DSM) where sources and sinks can be identified by DSM banding. In essence, the result is a simple, directed graphs with three sets of edge weights (i.e. embodied energy content, disassembly cost, and recycling value). To clarify, in this specific application, each source within the disassembly network represents a first-level disassembly step, in which a component can be directly dismantled from the machine itself with no prior steps. The sinks of the disassembly network are coined as "decision points", in which a component is assessed as a reasonable stopping point for the complete disassembly.

\section{Disassemblability Ranking Criteria}

Das et al. 2000 developed a multi-factor model to compute a disassembly effort index (DEI) score, which is representative of the total operating cost to disassemble a product. It should be noted that the actual cost of disassembly would vary among customers due to differences in labor costs, regional taxes, and other local economic effects. This further motivates implementing a qualitative scale for measuring disassemblability.

Figure 2 illustrates the scorecard with suggested ratings for each criterion. As can be seen, there are 7 different criteria that are individually weighted in terms of importance for disassembly cost. For example, the tools required to disassemble an object from its core is deemed about half as important as the force required to dismantle the component. Hence, the "tools" rating is out of 10 points while the "force" rating is set out of 20 points. The total DEI score adds up to 100 , where 0 equates to the simplest disassembly while a score of 100 denotes the toughest. It again should be noted that this specific methodology was developed specifically for PC (personal computer) components and the suggested values (e.g. $>210$ for 25/25 in "time") are based on heuristical knowledge in 


\begin{tabular}{|c|c|c|}
\hline \multirow{2}{*}{$\begin{array}{l}\text { 1. TIME } \\
\text { (Secs) }\end{array}$} & $>210$ & SCORE \\
\hline & $\left.\left.\left.\left.\left.\left.\right|_{25} 1111\right|_{20} 1111\right|_{15} 1111\right|_{10} 1111\right|_{5} 1111\right|_{0}$ & \\
\hline \multirow[t]{2}{*}{ 2. TOOIS } & improvised & SCORE \\
\hline & 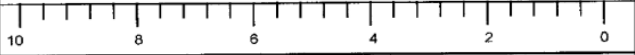 & \\
\hline \multirow[t]{2}{*}{ 3. FIXTURE } & Two-Hands & SCORE \\
\hline & $\left.\prod_{15}|1| 1||_{12}|1| 1||_{9} 1111||_{6}|1| 1||_{3}|1| 1\right|_{0} ^{1}$ & \\
\hline \multirow[t]{2}{*}{ 4. ACCESS } & Not Visibie Dual-axis & SCORE \\
\hline & 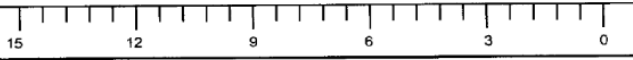 & \\
\hline \multirow[t]{2}{*}{ 5. INSTRUCT } & Training Contact OEM Group Discuss & SCORE \\
\hline & 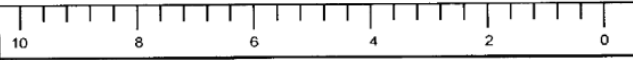 & \\
\hline \multirow[t]{2}{*}{ 6. HAZARD } & Fire Protection Face Mask/Arm wrap Gloves & SCORE \\
\hline & 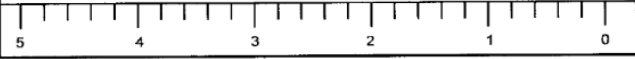 & \\
\hline \multirow[t]{2}{*}{$\begin{array}{l}\text { 7. FORCE } \\
\text { - Unfasten } \\
\text { - Human } \\
\text { - Machine }\end{array}$} & 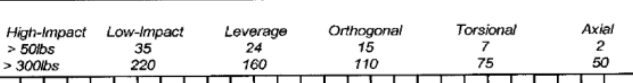 & SCORE \\
\hline & 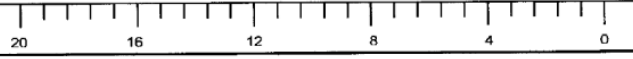 & \\
\hline
\end{tabular}

Figure 2: DEI scoring card [11]

the computer assembly industry. This suggested scale might not be applicable to heavy machinery; however, the general idea of the methodology serves its purpose. Project stakeholders, responsible for scoring were given the option to exceed the maximum criterion score provided. In order to derive the total disassembly effort, each sub-criterion score is normalized to its set weight (e.g. "time" was normalized to a score out of 25), providing a total normative score out of 100 points.

A deeper description of the seven criteria for the DEI score are provided below.

1) Time. The disassembly time includes set-up time, handling time and the actual active disassembly time. Because of the brevity of this specific project as well as the defining purpose and goal of the study, exact handling and disassembly times are not vital in order to achieve successful outcomes. This specific criterion was modified to be case-relevant. According to assembly floor engineers, for example, 210 seconds to disassembly even the simplest of subassemblies from the mining rig would be considered a very reasonable and short time.

2) Tools. Tools include all electromechanical as well as any handling devices needed to complete a disassembly step. Similar to assessing time for disassembly, tools required to dismantle subassemblies within the large operating drilling machines are quite different than PCs. As a result, a similar approach as criterion 1 is used within this ranking criterion.

3) Fixture. Since many disassembly operations are rather complex and require more than one hand to perform, means to fixture components prove as important considerations. Necessary clamps, winches, and necessary automation are included in the score.

4) Access. The ability to access hidden and obstructed components and subassemblies greatly affects the ease of disassembly of products as well. The score also includes how the item is removed (e.g. axially, laterally, or both).
5) Instruct. Similar to the assembly process, disassembly requires instruction from the perspectives of safety, efficacy, efficiency, and general knowhow. Since this report is aimed at educating clients for which subassemblies they should target for sellback, instruction becomes critical as an enabler for these plans to come into fruition. It should be noted that this criterion also includes how often the disassembly operators would have to contact the machine's distributer or the OEMs for specific information.

6) Hazard. The mining rig's manufacturer is a socially conscious company in which safety is their highest priority. This is stated throughout their mission statement and within a plethora of press releases and intra-company reports. Though the rig has no toxic chemicals associated with its design, oil from various parts (e.g. engine, compressor) provides some concern that should be included into the overall scoring of disassembly.

7) Force. The force required to dismantle certain components is quite significant. For example, within the various cylinders used throughout the rig for hydraulic powered mechanisms, the pistons/rods are pressure loaded within their respective tubes. To disassemble such components, it may be necessary to use heavy tools to deliver significant axial, torsional or orthogonal force.

Specific disassemblability scoring was conducted by interviewing design team members and assembly-floor employees with extensive experience with the components studied.

\section{Streamlined LCA}

A streamlined LCA was conducted per component to include an additional weight criterion for each edge (or link) within the network. Due to limited data availability across each component's lifecycle, only impacts related to material processing and recycling were included in the life cycle analysis. Though this specific study did not consider some fundamental stages of a product's lifecycle (e.g. manufacturing, assembly, distribution, use phase), the analysis still provides a snapshot of the relative importance of each component studied based on its material makeup. All impacts associated with recycling each respective material was taken into account through the Equation 1, where the total impact, both in terms of embodied energy and $\mathrm{CO} 2$ content, is calculated by the impact associated with producing the required amount of material per component minus the impact associated with recycling the same amount of material.

$$
\operatorname{Im} p_{\text {Total }}=\operatorname{Im} p_{\text {Primary Mat.Production }}-\operatorname{Im} p_{\text {Recycling }}
$$

All impacts were derived from Granta's material database, CES Edupack 2010® developed by Cambridge University, licensed through Purdue University [18]. This particular database provides conservative estimates of energy and $\mathrm{CO} 2$ outputs of primary material production, material 
processing (e.g. casting, forging, conventional machining) and material recycling. All impacts associated with database are quantified based on weight. For each entry from the Granta database, the average between the low and high estimates was used in the analysis (e.g. embodied energy for the primary production of cast iron was assumed to be $17.2 \mathrm{MJ}$, the average of 16.4 and 18.2). Since the system studied was of Scandinavian origin, material equivalency tables were used to build the life cycle inventory (LCI), as seen in Table 1. Each Granta CES Edupack material equivalent was assumed to be representative of the actual component's material as not to significantly affect the outcomes. Actual LCA results were omitted from this paper for confidentiality purposes.

Table 1: Material equivalency table

\begin{tabular}{l|l}
\hline Supplier Material & Granta CES Edupack 2010 Equivalent \\
\hline AISI 316 & Stainless steel, austenitic, AISI 316, wrought \\
AISI 329 & Stainless steel, duplex, AISI 329, wrought \\
AlZn5, 5Mg1 & Aluminium, 7075, wrought, T6 \\
Cast Iron & Cast iron, flake graphite BS grade 200 \\
CCR Rubber & Styrene butadiene rubber (SBR, 30\% black) \\
Fe510 D & Carbon steel, AISI 1022, normalized \\
Fe52 D & Carbon steel, AISI 1022, normalized \\
G24Mn6 & Low alloy steel, AISI 4140, normalized \\
General Plastic & ABS (medium-impact, injection molding) \\
General Steel & Low alloy steel, AISI 4140, normalized \\
Glass & Laminated glass \\
GS - AISI 10Mg, T6 & Aluminium, 354, cast, T6 \\
GS - Mn6 & Low alloy steel, AISI 4140, normalized \\
OVAKO 520 & Low alloy steel, AISI 4140, normalized \\
Polyethylene & PE-HD (general purpose) \\
S355J2+N & Carbon steel, AISI 1022, normalized \\
S355K2+N & Carbon steel, AISI 1022, annealed \\
SS 2142 & Stainless steel, duplex, AISI 329, wrought \\
St52 & Carbon steel, AISI 1022, normalized \\
\hline
\end{tabular}

For an additional edge weight, recycling value of each component was estimated via online material quotes.

\section{Dijkstra's Shortest Path Algorithm}

Once the disassembly network is defined, Dijkstra's shortest path algorithm is applied to find the minimum propagated disassembly cost across any given path (i.e. from a source node to a decision point). This specific algorithm is the most common method for traversing graphs with nonnegative edge path costs and has been well understood for decades [19]. Figure 3 illustrates a simple example of multiple pathways from a source node to a decision point. Each link has a specified weight associated with it. Optimal paths are identified by calculating a propagated path edge weight.

Eq. 2 shows that Dijkstra's algorithm simply discovers the shortest path by summing the edge weights across a specific path. If $P$ consists of edges $e_{0}, e_{1}, \ldots, e_{k-1}$ then the length of $P$, denoted $w(P)$, is defined as:

$$
w(P)=\sum_{i=0}^{k-1} w\left(e_{i}\right)
$$

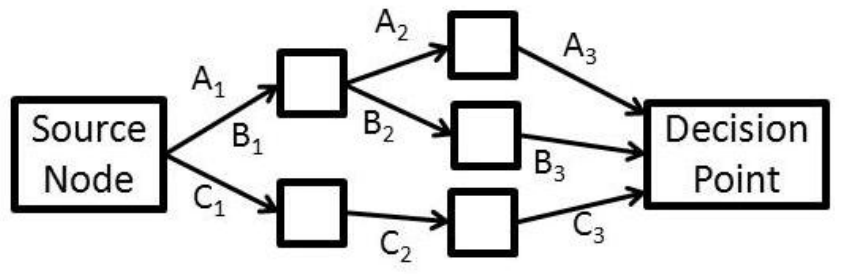

Figure 3: Schematic of three disassembly paths from one source node to a specific decision point.

In the case of Figure 3, for example, path $A$ would have the length equal to the sum of $A_{1}, A_{2}$ and $A_{3}$. It should be noted that it is possible for different paths to pass through the same intermediate nodes, as illustrated by $\mathrm{A}_{1}$ and $\mathrm{B}_{1}$.

Where the input is a simple undirected weighted graph $G$ with nonnegative edge weights, and a distinguished vertex $v$ of $G$ and the output is a labeled $[u]$, for each vertex $u$ of $G$, such that $D[u]$ is the distance from $v$ to $u$ in $G$.

In this specific case, three separate edge (or link) weights were included on the network to expand the decision space and discover the globally optimal scenario. The three edge weights were defined as (1) the difficulty of disassembly based on a component's DEI score, (2) the inverse of the reward embodied energy recovered, calculated by Eq. 1, and (3) the inverse of the reward recycling payback value. Normalized inverses of the reward weights are taken, because Dijkstra's algorithm treats weighted links as resistances, meaning the larger the weights the more difficult it is to traverse that specific edge. For clarity, the resultant is three identical disassembly networks with three unique edge weight values. The algorithm finds the shortest path from each source to all decision points across all three graphs.

\section{RESULTS}

The sequenced weighted disassembly graphs are implemented within Matlab®. Four sources for the disassembly graph were identified by sequencing the subsequent adjacency matrix, highlighted in orange in Figure 4. Sources, decision points, and network relationships are identical across all three separate disassembly graphs; however, the edge weights are distinct. Each feasible path is plotted, shown in Figure 5, based on the path's propagated disassembly effort and the sum of embodied energies of components within that path. It should be noted that the resultant graph presents a tradeoff decision. In theory, the most desirable disassembly plan would center on a path that has the lowest propagated disassembly effort and the highest recoverable energy, as illustrated by the 'desirable region' in Figure 5. However, it is unlikely for both parameters to be optimize in a single path; hence, a tradeoff problem is presented. In this case, many of the outputs of the shortest path algorithm across the networks are identical. Here, only embodied energy estimations were used to tradeoff against disassembly cost. One step further would implement other significant component characteristics (i.e. CO2 content, water usage, remanufacturing affinities) in order to align with specific organizational goals. 


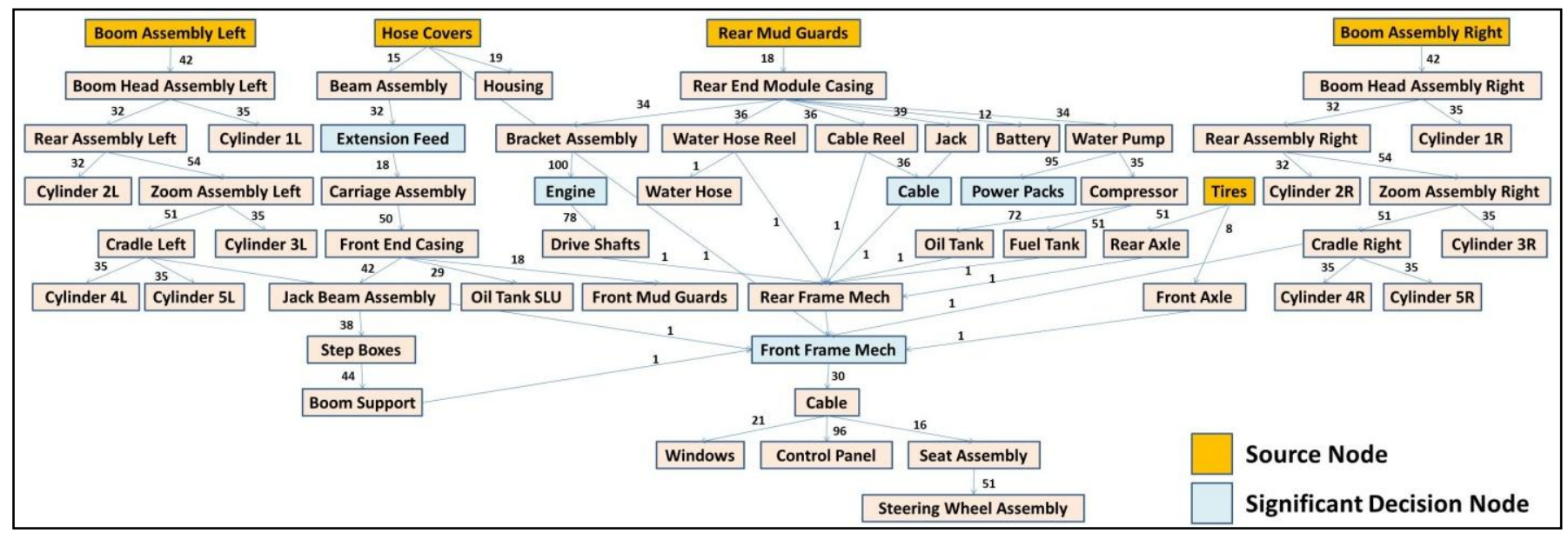

Figure 4: Representation of the resultant disassembly network with each component's respective normalized disassembly weights shown as edges. Each source node is represented by an orange-shaded box, while examples of significant decision nodes are shown in light blue.

As seen in Figure 5, there exits only two feasible paths in the 'desirable region'. The cables $(3,19)$ and power packs $(3,17)$, should be the primary candidates for recovery. Interestingly, the propagated disassembly effort score yields different results than using an un-propagated model, in which only single disassembly steps against each component's recovery value. This is due to disassembly sequencing and multiple steps may be necessary to expose a component of high value. It is also worth mentioning that components with high copper content (i.e. the cables and power packs) dominate the product's total embodied energy from a material perspective. In order to discovery all feasible disassembly paths, it might be worthwhile to investigate additional paths shown underneath the 'desirable region' in Figure 5. Scaling the y-axis differently might shift energy-intensive steel components, for example, with low disassembly effort into the feasible solution range. The product team ultimately responsible for these decisions should have a specific goal in terms of percent embodied energy recovered. The team can meet these goals by focusing on low propagated disassembly effort in order to ensure cost feasibility. Also, the project team can suggest specific redesigns

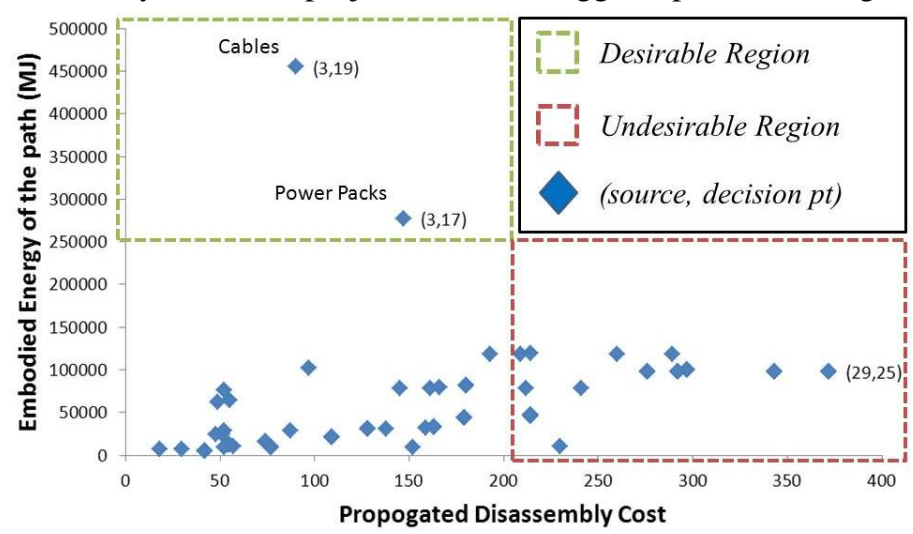

Figure 5: Plot of feasible paths with propagated disassembly effort and embodied energy recovered. of top-level components in order to augment energy-intensive components that, as of now, are within disassembly paths with very high propagated disassembly effort (i.e. within the 'undesirable region').

To finalize the suggested disassembly pathways, it is suggested to validate the disassembly cost with a more extensive, quantitative method to confirm its feasibility as well as calculate a more accurate project cost.

\section{CONCLUSIONS \& FUTURE DIRECTIONS}

This paper presents a general methodology for instituting initial material recovery plans for industrial machinery, for which dissembling entire high-level modules is unfeasible. The work aims to apply a disassembly assessment technique by comparing a component's disassembly effort to a reward such as recycling value or energy recovery from recycling. First, the disassembly network is represented by a directed graph where weighted edges represent reward/cost. Next, an implementation of Dijkstra's algorithm is used to compute the optimal disassembly path that minimizes the sum of the edge weights. Lastly, the optimal disassembly paths for each individual reward are compared to discover the globally optimal disassembly scenario. This method is applied to a realworld case study of an underground mining drill rig with direct contributions from engineers involved in the design of the machine itself. Specific component recovery options are recommended based on the methodology and alternative design practices are suggested to improve product recyclability.

Future directions include (1) investigating alternative graph transversal algorithms, (2) incorporating remanufacturing and reuse affinities into model in order to account for more of the EOL decision space, (3) implementing disassembly pathway recommendations with a machine rig's user and comparing actual disassembly effort/cost with the proposed model to validate methodology and (4) testing methodology on a complete network of components (e.g. all 5000 components in the given case study) for a product of similar complexity. 


\section{ACKNOWLEDGEMENTS}

This work was partially supported by TEKES, the Finnish Funding Agency for Technology and Innovation, as well as the National Science Foundation (NSF) under grant EEC-0935074. This paper does not necessarily reflect the view or opinions of either agency. The authors would like to thank the support of the Production Department at the Tampere University of Technology, including valuable insight from Prof. Seppo Torvinen. Also, the authors recognize Jouni Niemi, a student worker from Tampere University of Applied Sciences (TAMK), who conducted interviews with the design team members in regards to the disassemblability scoring and also gathered necessary information from suppliers to build the LCI for the LCA portion of the project.

\section{REFERENCES}

[1] K. Ramani, D. Ramanujan, W. Z. Bernstein, F. Zhao, J. Sutherland, C. Handwerker, J. K. Choi, H. Kim, D. Thurston, Integrated Sustainable Life Cycle Design: A Review, Journal of Mechanical Design, 132 (9), 2010.

[2] M. Fleischmann, J. M. Bloemhof-Ruwaard, R. Dekker, E. van der Laan, J. A. E. E. van Nunen, L. N. Van Wassenhove, Quantitative models for reverse logistics: A review, European Journal of Operational Research, 108, 1997, pp. 1-17

[3] M. A. Ilgin, S. M. Gupta, Environmentally conscious manufacturing and product recovery (ECMPRO): A review of the state of the art, Journal of Environmental Management, 91, 2010, pp. 563-591.

[4] S. K. Srivastava, Green supply-chain management: A state-of-the-art literature review, International Journal of Management Reviews, 9, 1, 2007, pp. 53-80.

[5] J. Reap, F. Roman, S. Duncan, B. Bras, A survey of unresolved problems in life cycle assessment, International Journal of Life Cycle Assessment, 13, 2008, pp. 374-388.

[6] F. Afrinaldi, M. Z. Mat Saman, The evaluation methods of disassemblability for automotive components - a review and agenda for future research, Journal Mekanikal, 26, 2008, pp. 49-62.

[7] R. Teunter, Determining optimal disassembly and recovery strategies, Omega, 34 (6), 2006, pp. 533-537.

[8] B. Willems, W. Dewulf, J. Duflou, End-of-life strategy selection: A linear programming approach to manage innovations in product design, International Journal of Production Engineering and Computers, 6 (7), 2004, pp. 36-43.

[9] H. Zhang, T. Kuo, A graph-based approach to disassembly model for end-of-life product recycling, Proceedings of the $19^{\text {th }}$ IEEE/CPMT International Electronics Manufacturing Technology Symposium, 1996, pp. 247-254.

[10]E. Kroll, T. Hanft, Quantitative Evaluation of Product Disassembly for Recycling, Research in Engineering Design, 1998, pp. 1-14.
[11]S. Das, P. Yedlarajiah, R. Maremdra, An approach for estimating the end-of-life product disassembly effort and cost, International Journal of Production Research, 38 (3), 2000, pp. 657-673.

[12] T. Kuo, Enhancing disassembly and recycling planning using life-cycle analysis, Robotics and ComputerIntegrated Manufacturing, 22 (5-6), 2006, pp. 420-428.

[13] Tang, Y., Zhou, M., Caudill, R. J., An integrated approach to disassembly planning and demanufacturing operations, IEEE Transactions on Robotics and Automation, 2001, 17(6), 773-784.

[14] Gonzalez, B., Diiaz, B. A., A bill of materials-based approach for end-of-life decision making in design for the environment, International Journal of Production Research, 2005, 43(10), 2071-2099

[15]P. Shrivastava, H. C. Zhang, J. Li, A. Whitely, Evaluating obsolete electronic products for disassembly, material recovery and environmental impact through a decision support system, Proceedings of the IEEE International Symposium on Electronics and the Environment, 2005, pp. 221-225.

[16] J. Yi, C. Chen, C. Gu, T. Fan, Analysis on product recycling profit based on Disassembly-Logic-Net (DLN) model, Proceedings of the $2^{\text {nd }}$ International Conference of Information Science and Engineering (ICISE), 2010, pp. 1001-1006.

[17]DSMweb.org. http://dsmweb.org. Accessed September 20, 2011.

[18] M. F. Ashby, D. Cebon, New Approaches to Materials Education - A Course, Cambridge University Engineering Department, March 2002.

[19]E. W. Dijkstra, A note on two problems in connexion with graphs, Numerische Mathematik, 1, 2959, pp. 269271. 